\title{
Effect of Barrier Height on Nuclear Fusion
}

\author{
G. S. Hassan ${ }^{1}$, A. Abd-EL-Daiem, and A. M. Mahmoud \\ ${ }^{1}$ physics Department, Assiut University, Assiut, Egypt \\ ${ }^{2}$ Physics Department, Sohag University, Sohag , Egypt
}

\begin{abstract}
The enhancement of sub-barrier fusion has been interpreted due to coupling between the relative motion and other degrees of freedom. The coupling gives rise to the distribution of fusion barriers and passage over the lowest barrier which is responsible for fusion enhancement at energies below the barrier. There are several orders of magnitude could be considered due to the tunneling through the barrier. The barrier height could be deduced from the measured cross section data for different energies, as well as using many empirical forms for incomplete and complete fusion of two massive nuclei. Firstly, we present a formula for barrier height $(O D E F F)$ and check, over wide ranges of interacting pairs the percentage agreement with those calculated or measured values for all pairs within $Z_{P} Z_{T} \leq 3000$. Secondly, the more recently measured excitation functions are studied using four models of nuclear forces, indicating that most of them can be used for wide energy range while the others failed to do so.We refer this notice to the theory deducing the model. For this, the 14 undertaken pairs recover the range $18 \leq Z_{P} Z_{T} \leq 1320$
\end{abstract}

Keywords: fusion barrier, ODEFF function, excitation, nuclear potential, WKB approximation PACS: $25.60 . \mathrm{Pj} ; 25.70 . \mathrm{Gh} ; 25.70 . \mathrm{Jj}$

\section{Introduction}

The sub-barrier fusion provides a method to test the nuclear potential on the inner side of the interaction barrier and to gain information on the influence of nuclear structure upon the behavior of nuclear matter and dynamics of nuclear reactions, specially for energies where penetrability effects are considered. The sub-barrier fusion cross section presents an unexpected enhancement, as compared with conventional models of tunneling through a one-dimensional penetration model, which successfully describes fusion above the Coulomb barrier.

For sub-barrier fusion of two massive nuclei, it was discovered that there was several orders of magnitude more than sub-barrier, could be accounted in terms of quantal tunneling through the fusion barrier. The quantum mechanical barrier penetration effects play a central role in near- and sub-barrier fusion reactions, where the fusion cross section has been vanished suddenly as the bombarding energy becomes less than the interaction barrier.

\subsection{Fusion Barrier}

The probability of fusion of two heavy ions at energies below their mutual interaction barrier, is defined by a barrier radius given due to different reasonable forms [1,2]as: $R_{\text {fus }}=r_{i}\left(A_{p}^{1 / 3}+A_{T}^{1 / 3}\right)$ where $\mathrm{r}_{\mathbf{i}}$ is referring to the height and extension of the barrier, and has a critical value [3] as $r_{\mathbf{c}}=1.3$. The total energy $V_{T}$ required for a specified reaction channel $[3,4,5,6]$ is related to barrier height $v_{B}$ by:

$V_{T}\left(R_{f u s}, L\right)=V_{B}+V_{L}=V_{C}+V_{N}+V_{L}$

where $V_{\boldsymbol{c}}, \boldsymbol{V}_{\boldsymbol{n}}$ and $\boldsymbol{V}_{\boldsymbol{L}}$ are the Coulomb, nuclear and centrifugal forces respectively. The motion of the binary system is then described by Schrödinger equation:

$$
\left[-\frac{\hbar^{2}}{2 \mu} \frac{d^{2}}{d R^{2}}+V(R)-E\right] \Psi(R)=0
$$


where $\mathbf{E}$ is the excitation energy in the center of mass system. In order for fusion reactions to occur, the barrier height $\boldsymbol{V}_{\boldsymbol{B}}$ created by the strong cancellation between the repulsive Coulomb force $\boldsymbol{V}_{\boldsymbol{C}}$ and the attractive nuclear interaction $\boldsymbol{V}_{\boldsymbol{n}}$ has to be overcome. The nuclear force may be used in different forms and also based on different interpretations for the attraction between nuclei. Four forms of them are in high range of usage, namely:

1-The proximity potential which is based [4] upon the liquid drop model:

$V_{n}(r)=\frac{b c_{P} c_{T} c_{s}^{\prime}}{r_{0}^{2}\left(c_{P}+c_{T}\right)} \phi\left[\frac{r-\left(c_{p}+c_{T}\right)}{b}\right]$

2-The unified model, which is based [4] on the collective model:

$V_{n}(r)=-D\left(F+\frac{s}{a}\right) \frac{R_{P T}}{r} e^{-S / a}$

3- Woods - Saxon form represents [5] that force as

$$
V_{n}(r)=-16 \pi a \gamma R_{d} /\left(1+\exp \left(r-R_{0}\right) / a\right)
$$

$\mathbf{R}_{\mathbf{d}}, \mathbf{R}_{\mathbf{0}}$ are the reduced and half density radius, a is the diffusivity and

$\gamma$ is the average value of both the projectile and target surface tension .

4- R. Bass potential[6], presented in terms of the liquid drop model as :

$$
\begin{gathered}
V_{n}\left(R_{f u s}\right)=4 \pi \delta d \frac{R_{P} R_{T}}{R_{P}+R_{T}} \exp \left(\frac{-S}{d}\right) \\
=a_{S} A_{P}^{1 / 3} A_{T}^{1 / 3} \frac{d}{R_{P T}} \exp \left(-\frac{R_{f u s}-R_{P T}}{d}\right),
\end{gathered}
$$

With $\mathrm{d}=1.35$ and $\delta$ are the diffusivity and the specific surface energy and $R_{P T}=R_{P}+R_{T}$ is the sum of the half-maximum density radii. Both of these potentials are actively used for various ranges of ion masses and excitation energies. The many degrees of freedom quantum tunneling which is often called macroscopic quantum tunneling was firstly treated by Dasso and ,Broglia [7,8], in which the tunneling degree of freedom ( the elastic or entrance channel ) couples to the internal degrees of freedom ( the transfer and inelastic channels ). The concept of the distribution of barriers can be easy visualized classically when one of the interacting nuclei is deformed; this results in a dependence of the fusion barrier height on the orientation of the deformed nucleus and leads to a continuous distribution of potential barriers which extends below and above the conventional coulomb barrier

\subsection{Fusion Cross Section}

The reaction cross section through a definite channel of an energy $\mathrm{E}$ has been given by WKB approximation as a summation over all penetrating partial waves [9].

$$
\sigma_{r e c}=\pi \lambda^{2} \sum_{l=0}^{\infty}(2 l+1) T_{l}(E) P_{l}(E)
$$

where $\lambda, T_{1}(E)$ and $P_{1}(E)$ are reduced De Broglie wave length of the incident ion, the transmission coefficient and the probability of penetration respectively . For fusion we assume $\sigma_{\text {rec }}=\sigma_{\text {fus }}$ and $P_{l} \approx 1$. The upper limit in the last equation becomes $l_{\max },[1]$ and $\sigma_{\text {fus }}$ reads 


$$
\sigma_{f u s}=\pi \hbar^{2} \sum_{l=0}^{l_{\max }} \frac{(2 l+1)}{1+\exp \left[\frac{2 \pi\left(V-E_{c m}\right)}{\hbar \omega}\right]}
$$

Where $\omega$ is the harmonic oscillator frequency or curvature parameter. A logarithmic form is given by Wong as

$$
\sigma_{f u s}(\mathrm{E})=\left(\hbar \omega \mathrm{R}^{2} / 2 \mathrm{E}\right) \operatorname{lin}\{1+\exp [(2 \pi / \hbar \omega)(\mathrm{E}-\mathrm{V} \boldsymbol{B}(\mathbf{r}))]\}
$$

A sharp cut-off approximation assumes that relative angular momentum $l$ smaller than a particular critical angular momentum $\boldsymbol{l}_{\boldsymbol{c}}$ contribute to complete fusion, while higher values of $\boldsymbol{l}_{\boldsymbol{f} \boldsymbol{u}}$ are associated with direct (peripheral) process [10]

$$
\sigma_{f u s}=\frac{\pi \hbar^{2}}{2 \mu E_{c m}}\left(l_{\text {fus }}+1\right)^{2}
$$

Heavy-ion-induced fusion reactions can be treated classically and the cross sections are decomposed into partial ones corresponding to orbital angular momentum. This approximation gives the fusion cross section [10] similar to that given by equation(8) replacing $\boldsymbol{l}_{\boldsymbol{m a x}}$ by $\boldsymbol{l}_{\boldsymbol{f} \text { us }}$ as shown in equation (9). When applying the form on the measured data from more recent references we can deduce the critical $\boldsymbol{l}_{\boldsymbol{c} r}$ values as :

$$
\sigma_{f u s}=\frac{\pi \hbar^{2}}{2 \mu E_{c m}}\left(l_{c r}+1\right)^{2}
$$

\subsection{Angular Momentum Limits}

The formed composite nucleus by the complete fusion will decay either by fission or by evaporation. The evaporation residues cross section will represent :

$$
\sigma_{E R}=\frac{\pi \hbar^{2}}{2 \mu E_{C M}}\left(l_{e r}+1\right)^{2}
$$

Where as, $l_{e r}=l_{E R}$ for $l_{\max }>l_{E R}$, or $l_{e r}=l_{\max }$ for $l_{\max }<l_{E R}, l_{E R}$ is the specific angular momentum at which the partial level width for fission is equal to that for evaporation. For $l, l_{\max }$ the real potential no longer has a pocket, and so the cross section formula, eq. (12) in Sharp Cut-Off approximation tends to be

$$
\begin{array}{r}
\sigma_{\text {fus }}=10 \prod \boldsymbol{R}_{\text {fus }}^{2}\left(1-\mathrm{v}_{\mathbf{B}} / \mathrm{E}_{\mathrm{cm}}\right)=\sigma_{\mathrm{g}}\left(1-\mathrm{v}_{\mathbf{B}} / \mathrm{E}_{\mathrm{cm}}\right) \\
\sigma_{\text {fus }} / \sigma_{\mathrm{g}}=-\mathrm{v}_{\mathbf{B}}\left(1 / \mathrm{E}_{\mathrm{cm}}\right)+1
\end{array}
$$

The linear relation $\left(\sigma_{\text {fus }}, 1 / \mathrm{E}_{\mathbf{c m}}\right)$ leads to extraction of fusion radius as the maximum distance at which fusion can take place. This form has been used for a long time to predict the compound nucleus formation cross-section and it is also commonly used for the heavy- ion fusion reaction (figs. 1.a,1.b).

\section{One Dimension Empirical Formula for Fusion (ODEFF)}

Many years ago, using an empirical model called elastic model given by Scalia, we tried to make check and extension[11] on the study of fusion excitation functions for wider range of energies as well as wider range of interacting pairs and found that it is more significant and simpler for use. Similarly, it is well known that the barrier height could be deduced, using recently measured data, as the slope of the linear plot of eq.(12) . When applying this method for some recently measured data we calculate [12] for $\mathrm{Li}^{6}+\mathrm{Sm}^{\mathbf{1 4 4}}$ a slope $=0.99$ and $\mathrm{v}_{\mathrm{b}}$ $=21.4 \mathrm{Mev}$ and [13] for $\mathrm{Ca}^{\mathbf{4 0}}+\mathrm{Sn}^{\mathbf{1 2 4}}$ a slope $=0.99$ and $\mathrm{v}_{\mathrm{b}}=111.1 \mathrm{Mev}$ (see figs.(1.a,1.b). In addition, many empirical forms were introduced to calculate the barrier height of sub-barrier fusion of two massive nuclei. A recently deduced empirical form for fusion barrier was given by Kumari and Puri [14] depending on two dimensions ( $\mathrm{Z}$ and $\mathrm{A}$ of the interacting pair) as :

$$
V_{B}(x)=\alpha x^{2}+\beta x+\gamma
$$

Where $\mathrm{X}=1.44 \mathrm{z} /\left(\mathrm{A}_{\boldsymbol{P}}{ }^{\mathbf{1 / 3}}+\mathrm{A}_{\boldsymbol{T}}{ }^{\mathbf{1 / 3}}\right), \boldsymbol{Z}=\boldsymbol{Z}_{\boldsymbol{P}} \boldsymbol{Z}_{\boldsymbol{T}}, \alpha=4.53 \times 10^{-\mathbf{4}}, \beta=0.93, \gamma=-1.01$

Our new form for barrier height is deduced by applying the least square method on wide range of measured data to give a function of one parameter $\boldsymbol{Z}$ only: 


$$
\mathrm{V}_{B}(Z)=\mathbf{a} Z^{3}+\mathbf{b} Z^{2}+\mathbf{c} Z+\mathbf{d}
$$

With $\boldsymbol{Z}=\boldsymbol{Z}_{\boldsymbol{P}} \boldsymbol{Z}_{\boldsymbol{T}}$ and $\mathrm{a}=2.926 \times 10^{-8}, \mathrm{~b}=2.479 \times 10^{-8}, \mathrm{C}=0.0641, \mathrm{~d}=9.706$.

Firstly, fig.(2.a,2.b), indicates that the two curves calculated by eq.(14.a) are similar to those calculated using $b=0$, which means that the second term could be neglected without any variation on the curve smoothing and equation (14.a) can be reduced to the form

$$
\mathbf{V}_{B}(Z)=\mathbf{a} Z^{3}+\mathbf{c} Z+\mathbf{d}
$$

for the range $\quad Z \leq 800$, while using $(a=b=0$. ), the form

$$
\mathbf{V}_{B}(Z)=\mathbf{C}+\mathbf{d}
$$

is the more applicable for the range $800<Z \leq 3000$ as we will check in the next section. We make the required comparisons using either the calculated or measured data. Three sets of data are taken in consideration for comparisons, the recently calculated barriers using both of unified or proximity nuclear forms, those calculated using Kumari and Puri empirical[14] form eq.(13 ), and those given by Ishiwara Dutt and R. K. Puri [15].

\section{Results and Discussion}

In this work, we deduce a new empirical function ( ODEFF function ) and make checks and normalization to be:

$$
\mathbf{v}_{B}(Z)=\mathbf{a} Z^{3}+\mathbf{b} Z^{2}+\mathbf{c} Z+\mathbf{d}
$$

where $\left(\boldsymbol{Z}=\boldsymbol{Z}_{\boldsymbol{P}} \boldsymbol{Z}_{\boldsymbol{T}}\right), \mathrm{a}=2.926 \times 10^{-8}, \mathrm{~b}=2.479 \times 10^{-8}, \mathrm{C}=0.0641, \mathrm{~d}=9.706$. For $\boldsymbol{Z} \leq \boldsymbol{8 0 0}$ and $\mathrm{a}=\mathrm{b}$ $=0, \mathrm{C}=0.0641, \mathrm{~d}=9.706$ for $800<\boldsymbol{Z} \leq \mathbf{3 0 0 0}$

In the table(1), we defined 14 studied pairs in addition to the corresponding $\boldsymbol{l}_{\boldsymbol{m a x}}$ values, while in figs.(1.a and 1.b) we make use for equation ( 12 ) to deduce the barrier height from excitation functions of the pairs taken from the corresponding references. The relations appear some agreement for the straight shape. The check of our deduced form with three sets of data are given in figs.(3.a,3.b) when comparing with those given by authors using both unified and R. Bass nuclear form but in figs.(4.a,4.b) it is clear that a higher agreement is found with calculated data using the two dimensional empirical form eq.(13). The final agreement and the best is that shown on figs. ( 5.a,5.b ) with those calculated by R. Bass model [15].

At energies near and above the Coulomb barriers, using four nucleus-nucleus interaction potentials, and three different forms for $\sigma_{\text {fus }}$ are employed eqs. $(8 . a, 8 . b, 9)$ in order to fit available measured fusion cross sections as shown in Table(1). It is found enhanced values in comparison with the 1D BPM predictions. It is found also that the degree of enhancement strongly depends on the type of the target nucleus; spherical or deformed. We found also, that the unified and R. Bass nuclear formula are the joker for fitting measured excitation functions near the barrier or even when exceeding up to twice its value. The unified potential points out successful predictions of data for the intermediate ion reactions through both formulas. On the other hand, the success of the proximity potential comes next to that of R. Bass and unified models under the same circumstances and poor fits with data are obtained by it. Also we found that when the charge product $\boldsymbol{Z}_{\boldsymbol{P}} \boldsymbol{Z}_{\boldsymbol{T}}$ has a large value (heavy nuclei reactions), the smooth cut-off approximation $(8 . a, 8 . b)$ is the best to successfully predict the experimental data. For light nuclei systems (relatively low charge product $\boldsymbol{Z}_{\boldsymbol{P}} \boldsymbol{Z}_{\boldsymbol{T}}$ ), both of the smooth cut-off approximation and Wong formulas produce reasonable predictions using either unified or R. Bass. On the other hand, the proximity potential through the smooth cut-off approximation successfully reproduces the experimental data. Predictions of the Bass potential reveal reasonable agreement with data. This result may resemble the significant difference in depth between this potential and the others and the low level agreement for some pairs may be interpreted as results of nature and strength of the couplings. Thus lies in the distribution of fusion barrier and the experimental determination of this distribution, which are major steps for understanding heavy-ion fusion. The results of fitting are shown on figs.(6.1-6.14). The last note will be the main point of interest for research in the preceding work to interpret the effect of barrier distribution on fusion excitation functions.

\section{Conclusion}

The ODEFF function has been checked and normalized to give the final form (14.a and 14.c ), is a simple and easier form. Also it is a very high accurate form predicting the barrier height of fusion for any $\mathrm{X}+\mathrm{Y}$ interacting pair. The cross sections at low energies then result from passage over the lower barriers rather than penetration through the single barrier. Information on the nature and strength of the couplings thus lies in the 
distribution of fusion barrier and the experimental determination of this distribution is a major step towards understanding heavy-ion fusion. The last note will be the main point of interest for research in the preceding work to interpret the effect of barrier distribution on the excitation functions for fusion.

[1]. L C Vaz, et al, Phys. Rep., 69, 373, 1981

\section{References}

[2]. L C Vaz and J. M. Alexander, Phys. Rev. ,C 18 ,2152, 1978

[3]. R Bass, Nucl.Phys. ,A231 ,45, 1974

[4]. H J Krappe, et al, Phys .Rev. , C 20, 992, 1979

[5]. $\quad$ H Esbensen et al., Phys. Rev. , C 40 ,2046, 1989

[6]. R Bass, Phys. Rev. Lett. , 30, 265, 1977

[7]. C H Dasso et al , Nucl. Phys. , A405 381, 1983 and C H Dasso et al Nucl. Phys. , A407, 221, 1983

[8]. R A Broglia et al, Phys. Rev. C27, 233, 1983

[9]. M Hugi, et al, Nucl. Phys. , A368, 173, 1981

[10]. A M Stefanini, et al, Nucl. Phys. , A548 453,1992

[11]. G S Hassan, et al, Acta Phys. Pol. , B31(8),1799,2000

[12]. P K Rath et al , phys. Rev. , C79 , 51610,2009

[13]. J J Kolata, et al , phys. Rev. , C85 , 054603,2012

[14]. R Kumari and R. Puri, Nucl. Phys. , A993,135, 2015

[15]. Ishiwara Dutt and R K Puri, ,(http://arxiv.org/abs/1005.5213),2010

[16]. P A De Young,et al, Phys. Rev., C28,692,1983

[17]. L C Dennis, et al, Phys. Rev. , C26,981,1982

[18]. D G Kovar,et al, Phys. Rev. , C20,1305,1979

[19]. Y D Chan,et al, Nucl. Phys. , A303,500,1978

[20]. R M Anjos,et al, Phys. Rev. , C42,354,1990

[21]. P A DeYoung, et al, Phys. Rev. , C26,1482,1982

[22]. V K C Cheng, et al, Nucl. Phys., A322 168,1979

[23]. S Gary, et al, Phys. Rev. , C25,1877,1982

[24]. W Scobel, et al, Phys. Rev. , C14,1808,1976

[25]. M Hugi, et al, Nucl. Phys. , A368, 173,1981

[26]. C R Morton, et al, Phys. Rev. Lett. , 72, 4074, 1994

[27]. $\quad N$ Knyazheva et al, Phys. Rev. , C75 064602,2007

[28]. J Khuyagbaatar et al. Phys.Rev. C86 (2012) 064602

Table(I) Barrier radius $\mathbf{R}_{\text {fus }}(\mathbf{f m})$ and heights $\mathbf{V}_{\mathbf{B}}(\mathbf{M e v})$ and maximum angular momentum of the concerned interacting pairs. In the three columns it is given the nuclear model used to fit, fig. number and reference for measured data.

\begin{tabular}{|c|c|c|c|c|c|c|c|c|c|c|}
\hline Pair & $Z_{\mathrm{P}} \mathrm{Z}_{\mathrm{T}}$ & $\begin{array}{c}\mathbf{R}_{\text {fus }} \\
(\mathbf{f m})\end{array}$ & $\begin{array}{c}V_{B} \\
\text { Proximity }\end{array}$ & $V_{\boldsymbol{B}} \mathrm{W}-\mathrm{S}$ & $\begin{array}{c}V_{\boldsymbol{B}} \\
\text { Unified }\end{array}$ & $\begin{array}{c}V_{\boldsymbol{B}} \\
R . \text { Bass }\end{array}$ & $\begin{array}{c}l_{\text {max }} \\
\text { used }\end{array}$ & $\begin{array}{c}\text { Model } \\
\text { Fig. }\end{array}$ & Ref \\
\hline${ }^{10} \mathrm{~B}+{ }^{14} \mathrm{~N}$ & 35 & 7.60 & 6.15 & 5.69 & 5.78 & 6.55 & 12 & R.Bass & 6.1 & 16 \\
\hline${ }^{6} \mathrm{Li}+{ }^{12} \mathrm{C}$ & 18 & 7.12 & 3.29 & 3.02 & 3.03 & 3.59 & 9 & R.Bass & 6.2 & 17 \\
\hline${ }^{12} \mathrm{C}+{ }^{12} \mathrm{C}$ & 36 & 7.61 & 6.31 & 5.84 & 5.99 & 6.73 & 12 & R.Bass & 6.3 & 18 \\
\hline${ }^{12} \mathrm{C}+{ }^{16} \mathrm{O}$ & 48 & 7.84 & 8.23 & 7.65 & 7.80 & 8.71 & 13 & Unified & 6.4 & 19 \\
\hline${ }^{12} \mathrm{C}+{ }^{19} \mathrm{~F}$ & 54 & 8.03 & 9.08 & 8.46 & 8.63 & 9.58 & 14 & R.Bass & 6.5 & 20 \\
\hline${ }^{14} \mathrm{~N}+{ }^{14} \mathrm{~N}$ & 49 & 7.85 & 8.40 & 7.80 & 7.66 & 8.88 & 13 & R.Bass & 6.6 & 21 \\
\hline${ }^{16} \mathrm{O}+{ }^{16} \mathrm{O}$ & 64 & 8.07 & 10.74 & 10.01 & 10.24 & 11.30 & 15 & Unified & 6.7 & 22 \\
\hline${ }^{24} \mathrm{Mg}+{ }^{24} \mathrm{Mg}$ & 144 & 8.75 & 22.53 & 21.14 & 21.76 & 32.47 & 20 & Unified & 6.8 & 23 \\
\hline${ }^{27} \mathrm{Al}+{ }^{35} \mathrm{Cl}$ & 221 & 9.23 & 32.86 & 35.07 & 31.89 & 34.14 & 25 & Unified & 6.9 & 24 \\
\hline${ }^{28} \mathrm{Si}+{ }^{9} \mathrm{Be}$ & 56 & 8.26 & 9.18 & 8.61 & 8.76 & 9.66 & 14 & R.Bass & 6.10 & 25 \\
\hline${ }^{16} \mathrm{O}+{ }^{144} \mathrm{Sm}$ & 496 & 10.76 & 63.54 & 60.33 & 62.31 & 65.81 & 29 & R.Bass & 6.11 & 26 \\
\hline${ }^{64} \mathrm{Ni}+{ }^{96} \mathrm{Zr}$ & 1120 & 11.17 & 135.34 & 349.27 & 133.67 & 142.33 & 44 & R.Bass & 6.12 & 10 \\
\hline${ }^{48} \mathrm{Ca}+{ }^{154} \mathrm{Sm}$ & 1240 & 11.61 & 143.70 & 664.90 & 142.61 & 151.50 & 43 & Unified & 6.13 & 27 \\
\hline${ }^{36} \mathrm{~S}+{ }^{206} \mathrm{~Pb}$ & 1312 & 11.83 & 149.27 & 621.23 & 147.86 & 157.41 & 39 & Unified & 6.14 & 28 \\
\hline \hline
\end{tabular}




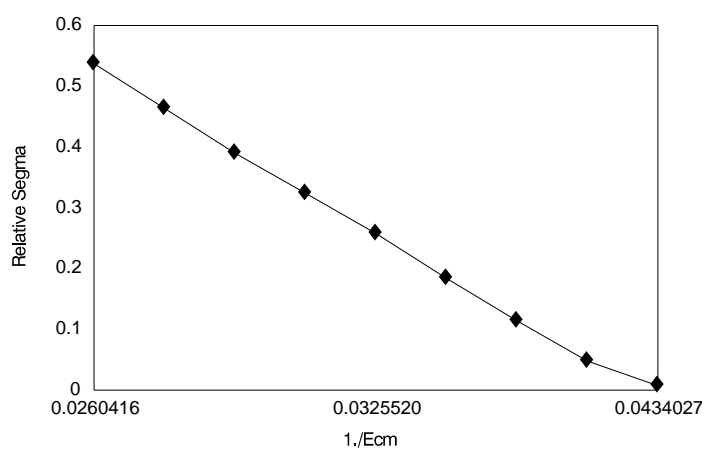

(1.a)

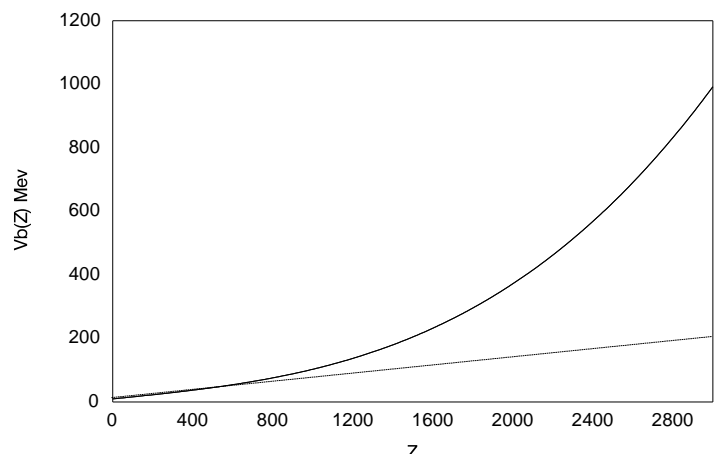

(2.a)

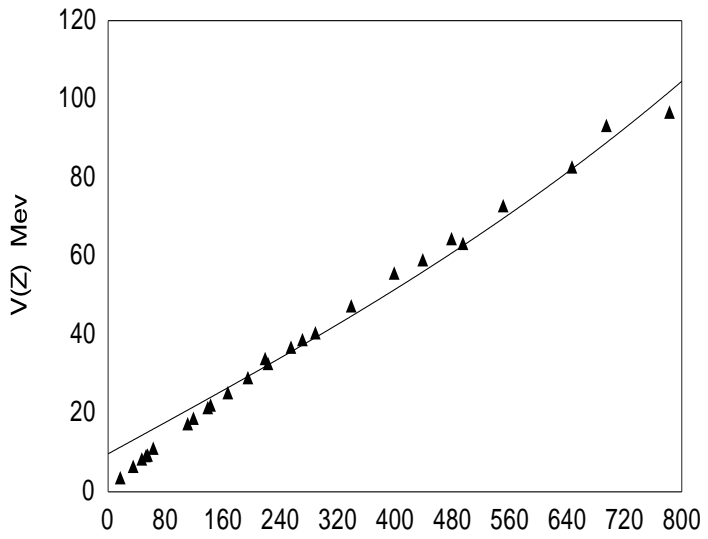

$\mathrm{Z}=\mathrm{ZpZt}$

(3.a)

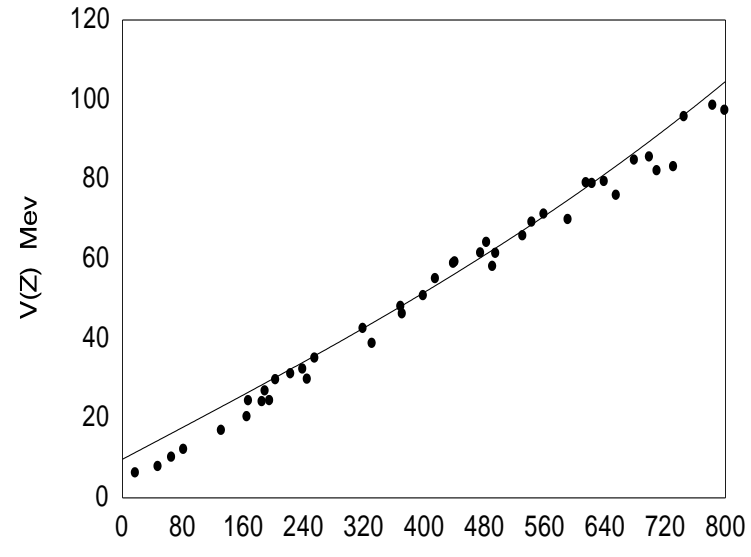

$\mathrm{Z}=\mathrm{ZpZt}$

(4.a)

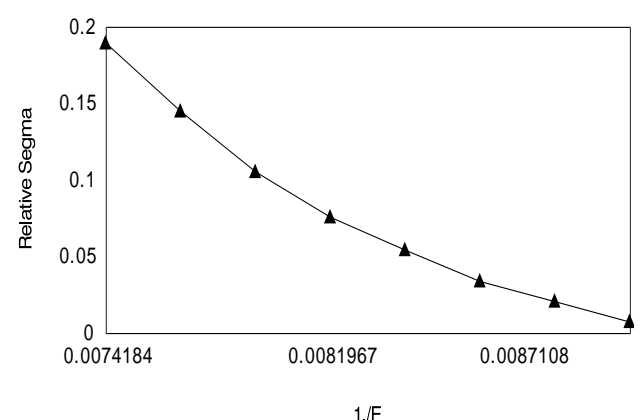

(1.b)

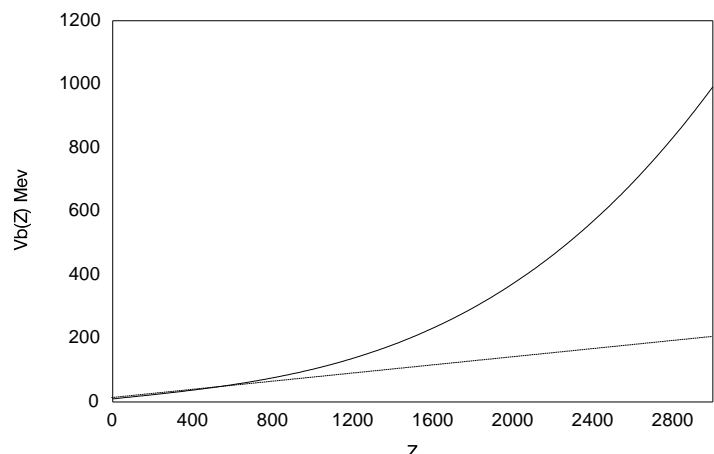

(2.b)

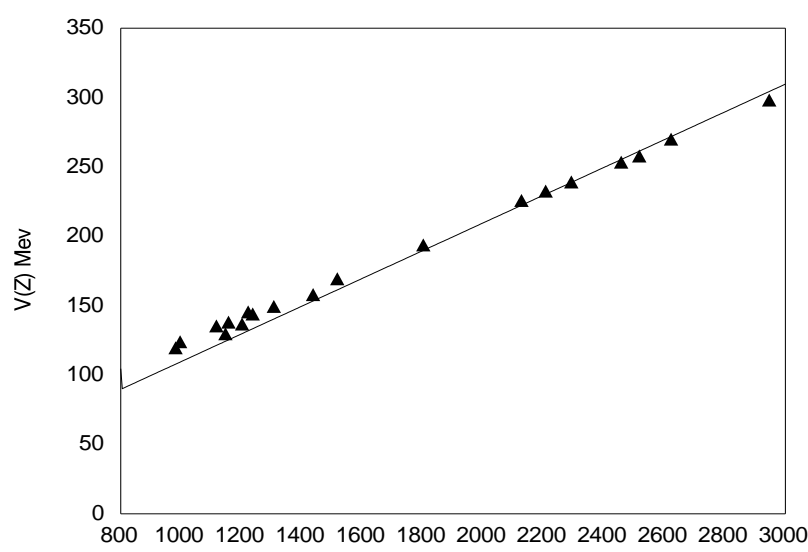

$\mathrm{Z}=\mathrm{ZpZ}$

(3.b)

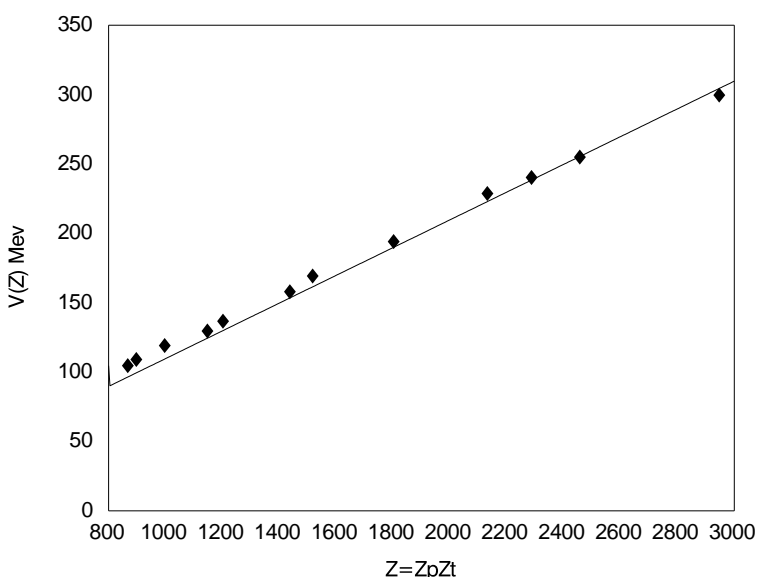

(4.b) 


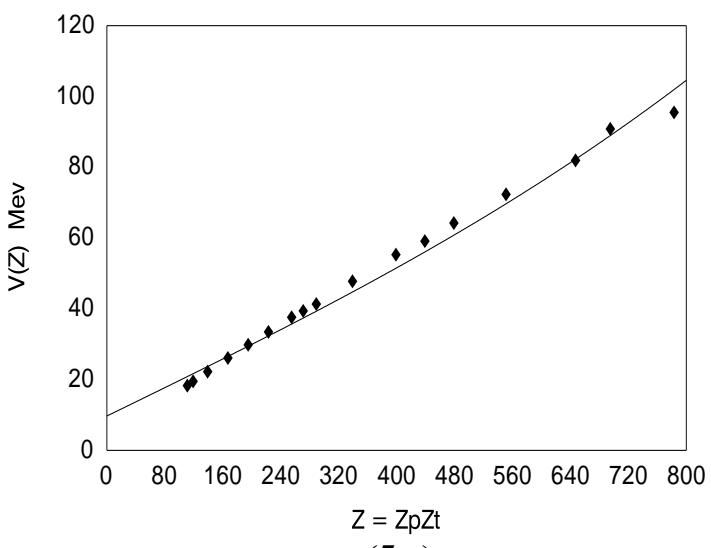

(5.a)

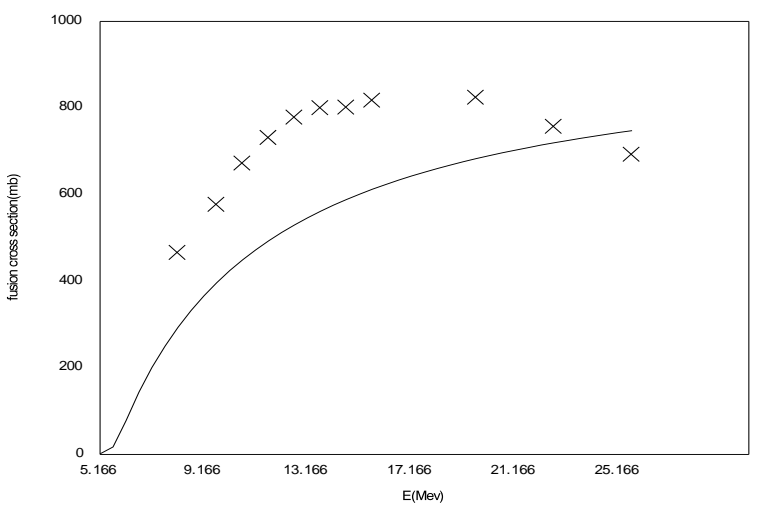

(6.1)
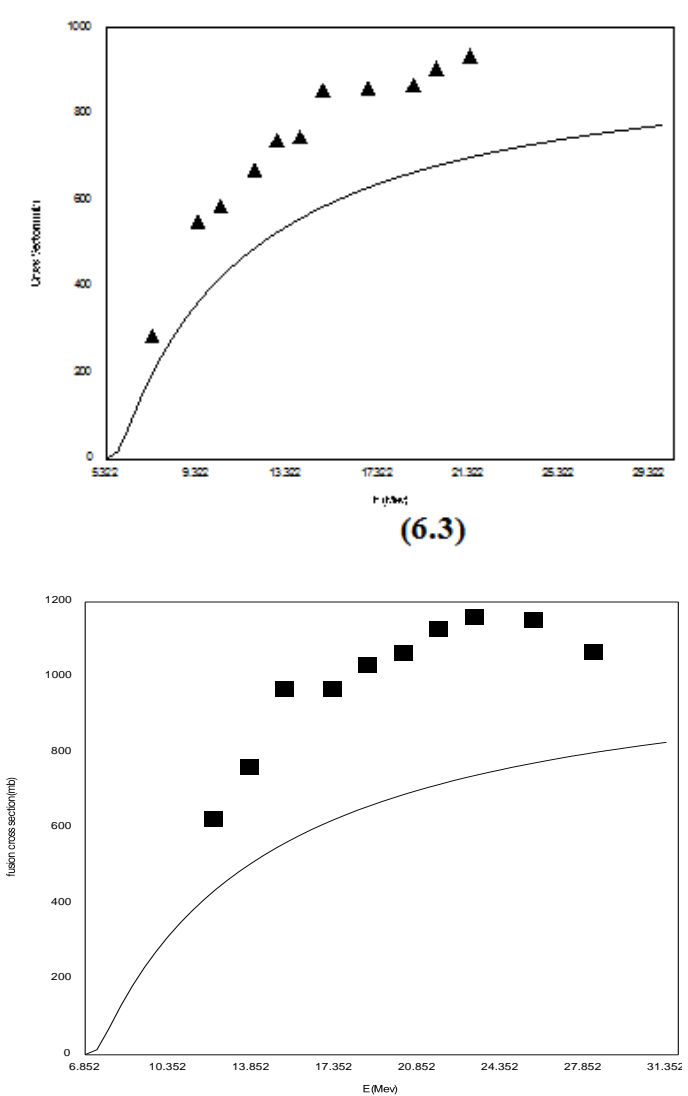

(6.5)

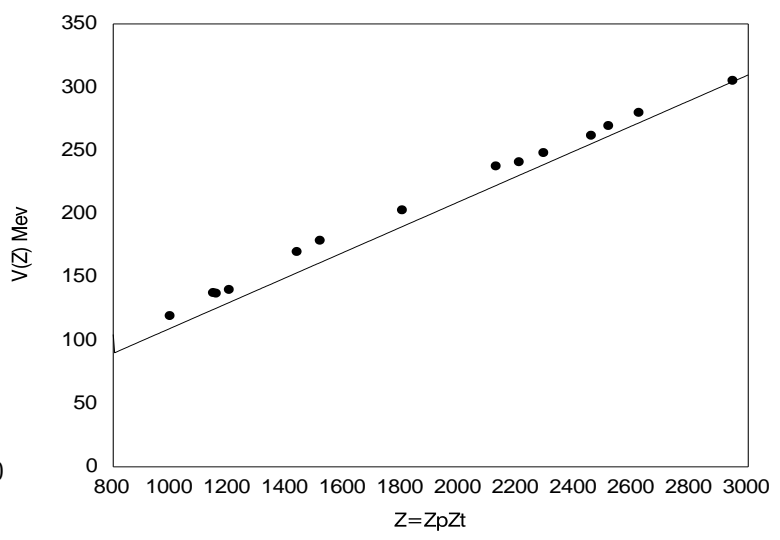

(5.b)

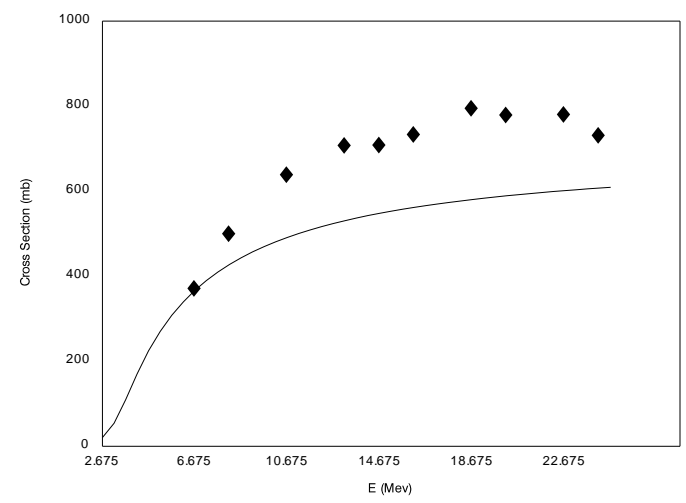

(6.2)
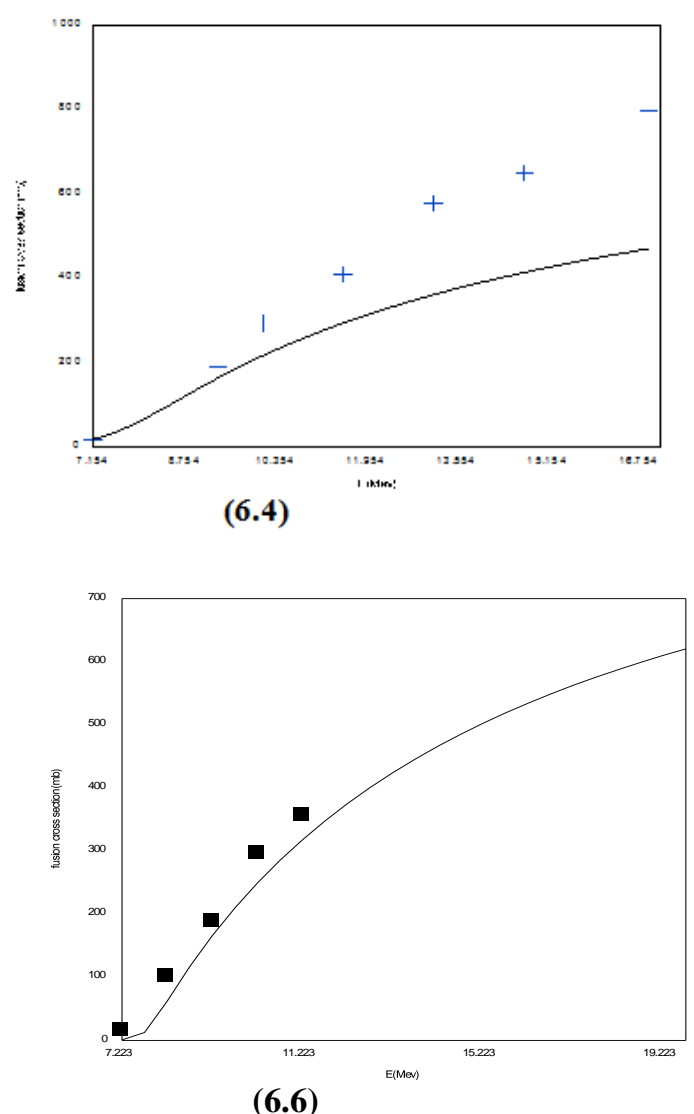


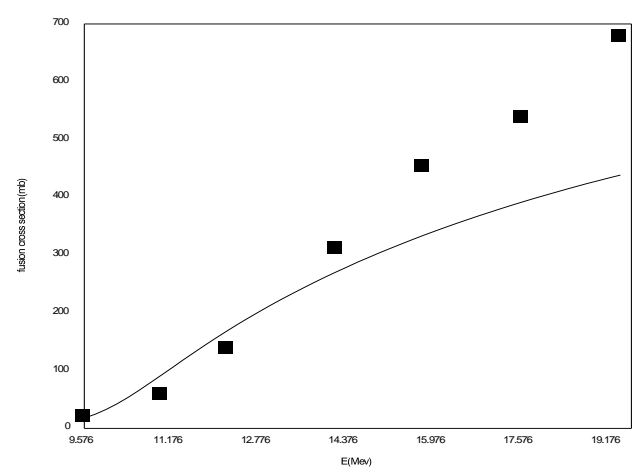

(6.7)

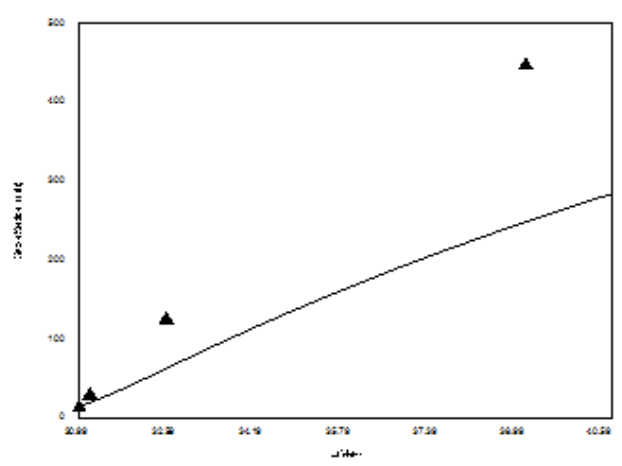

(6.9)
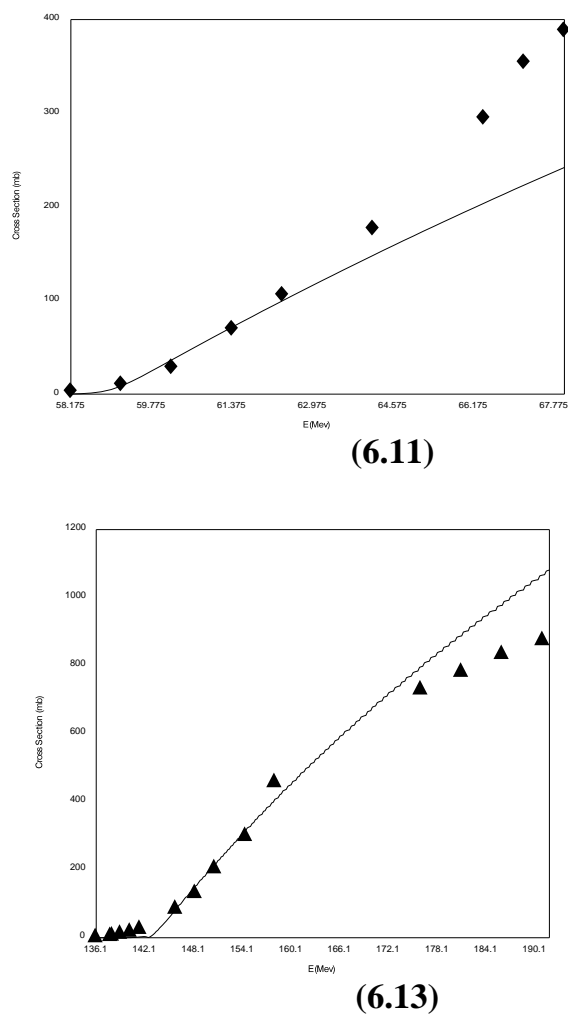

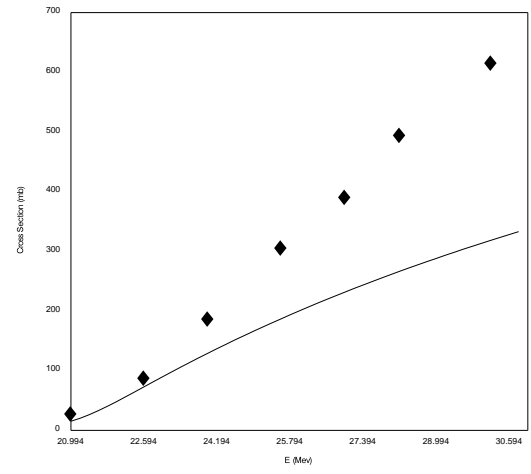

(6.8)

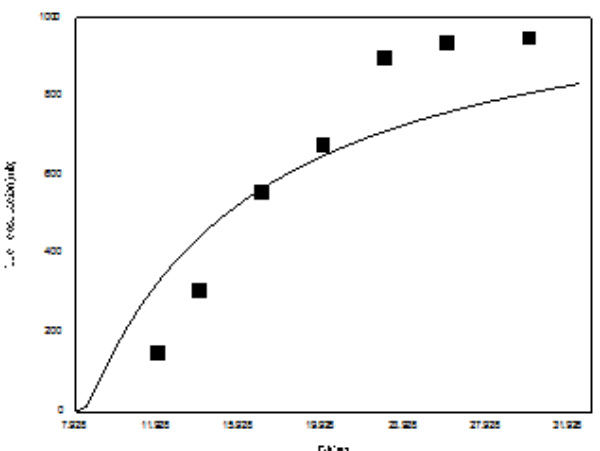

(6.10)

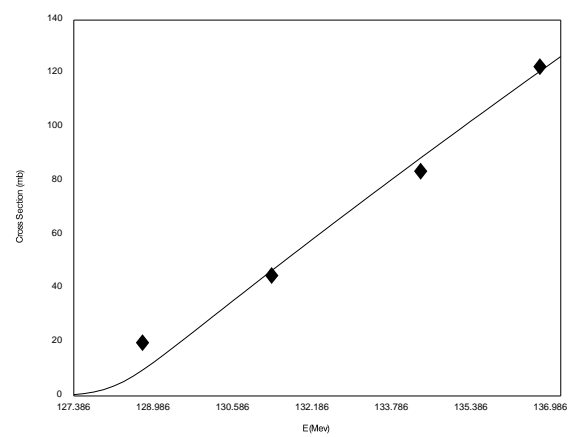

(6.12)

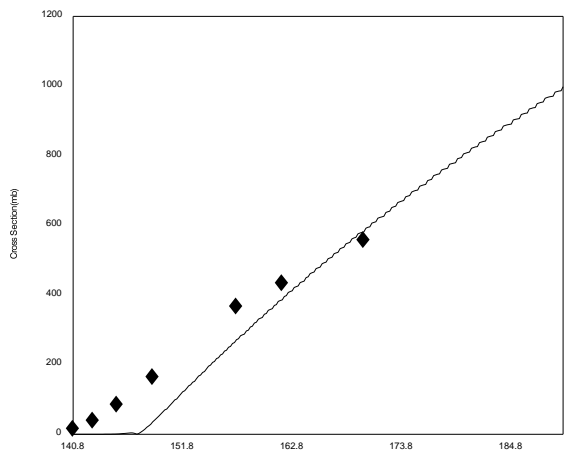

(6.14)

Figure Caption

Fig(1) linear representation $\left(1 / \mathrm{E}_{\mathrm{CM}}, \sigma_{\mathrm{Fus}}{ }_{\mathrm{G}} \sigma\right):$ (a) for $\mathrm{Li}^{6}+\mathrm{Sm}^{144}$ with slope $=0.99$ and $\mathrm{v}_{\mathrm{b}}=21.4 \mathrm{Mev}$, the data are taken from [12]

(b) for $\mathrm{Ca}^{40}+\mathrm{Sn}^{124}$ With slope $=0.99$ and $\mathrm{v}_{\mathrm{b}}=111.1 \mathrm{Mev}$, the data are taken from [13] 
Fig (2) The one dimensional empirical formula for fusion ODEFF function for $(0<Z<3000)$ : (a) is the form (14.a) in comparison with those calculated by eq.(14.c) (b) is the form (14.b) in comparison with those calculated by eq.(14.c)

Fig (3) Barrier height by ODEFF function, in comparison with those calculated using unified nuclear potential or proximity nuclear form (a) eq.(14.a) for $\boldsymbol{Z} \leq \mathbf{8 0 0}$ and (b) eq.(14.c) for $\mathbf{8 0 0}$, $\boldsymbol{Z} \leq \mathbf{3 0 0 0}$

Fig (4) Barrier height by ODEFF function in comparison with those calculated using the empirical [14] form, eq.(13) given by R. Kumari and R. Puri, (a) eq.(14.a) for $\boldsymbol{Z} \leq \boldsymbol{8 0 0}$ and (b) eq.(14.c)for 800 、 $\boldsymbol{Z} \leq \mathbf{3 0 0 0}$

Fig (5) Barrier height by ODEFF function in comparison with those calculated by Dutt and Puri using Bass 80 [15] form eq.( 1, 3 ) (a) eq.(14.a) for $\boldsymbol{Z} \leq \boldsymbol{8 0 0}$ and (b) eq.(14.c)for 800 、 $\boldsymbol{Z} \leq \mathbf{3 0 0 0}$

Figs (6.1-6.14) Calculated excitation functions for undertaken pairs in comparison with measured data (Table 1). The unified and R. Bass nuclear formula are the joker for fit near and above up to twice the barrier. When $Z_{P} Z_{T}$ has a large value, the smooth cut-off approximation is the best for successful predictions. For low charge product $Z_{P} Z_{T}$, both of the smooth cut-off approximation and Wong formulas produce reasonable predictions. On the other hand, the proximity potential and smooth cut-off approximation, fits successfully the experimental data. The low level agreement for some pairs may be interpreted as results of nature and strength of the couplings. 